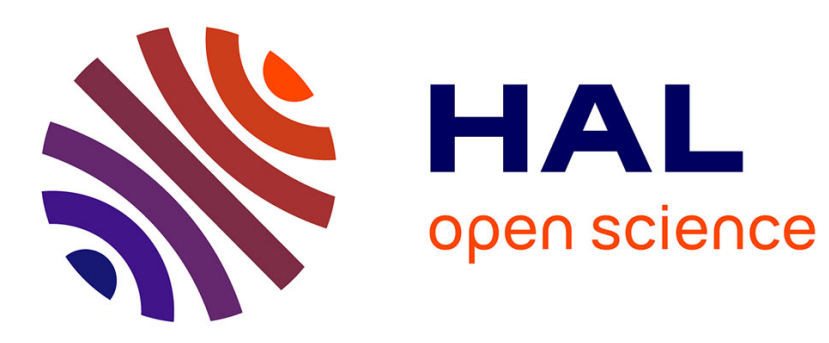

\title{
Families of Monotonic Trees: Combinatorial Enumeration and Asymptotics
}

\author{
Olivier Bodini, Antoine Genitrini, Mehdi Naima, Alexandros Singh
}

\section{To cite this version:}

Olivier Bodini, Antoine Genitrini, Mehdi Naima, Alexandros Singh. Families of Monotonic Trees: Combinatorial Enumeration and Asymptotics. 15th International Computer Science Symposium, Jun 2020, Yekaterinburg, Russia. pp.155-168, 10.1007/978-3-030-50026-9_11 . hal-03033670

\section{HAL Id: hal-03033670 https://hal.sorbonne-universite.fr/hal-03033670}

Submitted on 1 Dec 2020

HAL is a multi-disciplinary open access archive for the deposit and dissemination of scientific research documents, whether they are published or not. The documents may come from teaching and research institutions in France or abroad, or from public or private research centers.
L'archive ouverte pluridisciplinaire HAL, est destinée au dépôt et à la diffusion de documents scientifiques de niveau recherche, publiés ou non, émanant des établissements d'enseignement et de recherche français ou étrangers, des laboratoires publics ou privés. 


\title{
Families of Monotonic Trees: Combinatorial Enumeration and Asymptotics ${ }^{\star}$
}

\author{
Olivier Bodini ${ }^{1}$, Antoine Genitrini ${ }^{2}$, Mehdi Naima $^{1}$, and Alexandros Singh ${ }^{1}$ \\ 1 Université Sorbonne Paris Nord, Laboratoire d'Informatique de Paris Nord, CNRS, \\ UMR 7030, F-93430, Villetaneuse, France. \\ \{Olivier.Bodini, Mehdi.Naima, Alexandros.Singh\}@lipn.univ-paris13.fr \\ 2 Sorbonne Université, CNRS, Laboratoire d'Informatique de Paris 6 -LIP6- UMR \\ 7606, F-75005 Paris, France. Antoine.Genitrini@lip6.fr
}

\begin{abstract}
There exists a wealth of literature concerning families of increasing trees, particularly suitable for representing the evolution of either data structures in computer science, or probabilistic urns in mathematics, but are also adapted to model evolutionary trees in biology. The classical notion of increasing trees corresponds to labeled trees such that, along paths from the root to any leaf, node labels are strictly increasing; in addition nodes have distinct labels. In this paper we introduce new families of increasingly labeled trees relaxing the constraint of unicity of each label. Such models are especially useful to characterize processes evolving in discrete time whose nodes evolve simultaneously. In particular, we obtain growth processes for biology much more adequate than the previous increasing models. The families of monotonic trees we introduce are much more delicate to deal with, since they are not decomposable in the sense of Analytic Combinatorics. New tools are required to study the quantitative statistics of such families. In this paper, we first present a way to combinatorially specify such families through evolution processes, then, we study the tree enumerations.
\end{abstract}

Keywords: Analytic Combinatorics · Asymptotic enumeration · Increasing trees · Monotonic trees - Borel transform - Evolution process

\section{Introduction}

An increasing tree is a rooted tree whose nodes are labeled by integers in $\{1, \ldots, n\}, n$ being the number of nodes in the tree. Furthermore, each label appears exactly once and, along each branch, the sequence of labels is strictly increasing. Families of such increasing trees have been the subject of many studies, owing to their wide applicability to representing data structures in computer science, probabilistic urn models in mathematics, and evolutionary trees in biology.

For example, in the analysis of algorithms and data structures, the study of increasing trees is useful in understanding the typical behavior of heaps and

\footnotetext{
* This work was also supported by the ANR projects Metaconc ANR-15-CE40-0014.
} 
search trees (see [7]). In the study of permutations, increasing trees found usage in illuminating the behavior of local order patterns in permutations (see, for example, [6]). In biology, increasing trees find application as models of phylogenetic trees which, apart from encoding the relations between species, also encode temporal information in a way such that it encodes the history of some evolutionary process (see [5]). For a detailed and generic analysis of families increasing trees, see [1. A study of combinatorial differential equations related to various enumerative aspects of increasing trees, including path length enumeration for general increasing trees and enumeration of enriched increasing trees with respect to node height is presented in [11].

Increasingly labeled tree structures have also been studied in [15] under the guise of monotone functions of tree structures. These are mappings $f$ from the nodes of a tree $t$ to the set $\{1, \ldots, k\}$ such that if $a_{i}$ is the child of $a_{j}$, then $f\left(a_{i}\right) \geq f\left(a_{j}\right)$. The authors studied this labelling on t-ary, plane and non-plane trees. Other authors studied this scheme on different tree models like Motzkin trees in [2]. The typical shapes of these trees have been studied in [10[12]. A good summary can be found in the thesis presented in [13]. Note that, unlike the case of increasing trees, this model allows labels of $\{1, \ldots, k\}$ to appear any number of times, including zero times.

A related model, rooted increasing $m$-ary trees with label repetitions, also allowing multiple nodes to have the same label, appeared in [4] and can be seen as the foundations of our following new study that widely extends the latter model. These models of increasing trees are related to evolution processes in discrete time: starting from a single leaf, a tree is grown by selecting at each time-step a leaf and replacing it by an internal node to which new leaves are attached. By allowing at each step multiple leaves to be expanded in parallel, the authors thus obtain trees with label repetitions. Interpreting the evolution process they establish functional equations satisfied by the enumerating series of their model. But these series are purely formal: their radius of convergence is 0 , thus the use of direct analytic methods to solve the equations is non-viable. For the analysis of the series, an approximate Borel transform is used and then arguments based on the asymptotics of certain differential equations give the asymptotic behavior for the tree enumeration.

In our work we extend the study of [4], presenting a generic framework for an even more general class of combinatorial structures; in particular by relaxing the restrictions on node degrees (more precisely node out-degree in graph theory) and by allowing also for weakly increasing labeling sequences along branches of the trees. We study the following broad classes:

- Strictly monotonic trees: rooted trees $T$ whose internal nodes are labeled with integers such that the root is labeled by 1 and along each branch the sequence of labels is strictly increasing. We also require that if $\ell$ is the greatest label in $T$, then all integers from 1 to $\ell$ also appear as labels of some internal nodes. Finally, we take the size of a tree to be its number of leaves.

- Monotonic trees: these are as above, except that in this case we allow for weakly increasing sequences of labels along each branch. 
The plan of the paper is as follows. We conclude this section with the formalization of our problem and the exact statement of the results of the paper. Section 2 is then dedicated to the presentation of a number of applications in our framework. In Section 3 we present a detailed discussion of the combinatorial and asymptotic properties of the model we have quickly described above. This includes the derivation of a recurrence relation for such families of trees and a general asymptotic analysis of the recurrence relation. We then conclude the paper with a discussion of open problems and potential future directions.

To formalize the previous description, we recall the notion of a degree function, (following [1]), which in our case describes the tree evolution.

Degree function. We define a degree function to be a power series of the form 3 $\phi(z)=\sum_{i \geq 1} \phi_{i} z^{i}$. Combinatorially they are interpreted in one of two following ways, depending on how we see the integer non-negative values $\phi_{i}$. Firstly, we can interpret $\phi_{i}, i \geq 2$, as the number of possible colors of a node of degree $i$. In this context the objects of study will correspond to the aforementioned strictly monotonic trees (these also include the so-called weakly increasing trees of [45]). Alternatively, the coefficients $\phi_{i}, i \geq 2$, can be seen as the number of trees with $i$ leaves belonging to some class of plane rooted unlabeled trees (in the sequel, we will refer to elements of such classes as tree-shapes). In this second context the objects that we will construct are monotonic trees as defined above. In both contexts, $\phi_{1}$ must be interpreted carefully, owing to the definition of the evolutionary process below: $\left(\phi_{1}-1\right)$ corresponds either to the number of colors for unary nodes or to the number of unlabeled trees of size 1 in the corresponding plane rooted unlabeled tree class. Using this notion of a degree function we can now define the following evolution process.

Evolution process. Given some degree function $\phi$ with $\phi_{1}=1$, the following evolution process generates a strictly monotonic tree. The process starts at timestep 0 with a single leaf and at each time-step $i \geq 1$ is as follows:

1. Choose a non-empty subset $L$ of leaves of the so-far built tree.

2. For each leaf $\ell \in L$ choose an admissible degree and color $(r, c), r>1, \phi_{r}>0$ and one of its colors $1 \leq c \leq \phi_{r}$.

3. Replace each leaf $\ell$ with an internal node labeled by $i$ with color $c$ and having $r$ new leaves attached to it.

In order to generate monotonic trees, in which case the coefficients of $\phi(z)$ are alternatively interpreted as enumerating tree-shapes rather than node colors, a slight modification of the above process is required: at each iteration step $i$, each selected leaf is replaced by a tree-shape, rather than a colored internal node, and all internal nodes of this tree-shape are labeled by $i$.

Remark 1. If unary nodes are allowed, i.e we have $\phi_{1}>1$, at the end of each iteration step we can choose any subset of the unselected leaves (the ones that were present at the previous step and not newly-added during the current step) and expand each into a unary node with the desired color/shape.

\footnotetext{
${ }^{3}$ We take $\phi_{0}=0$ in anticipation of our model.
} 


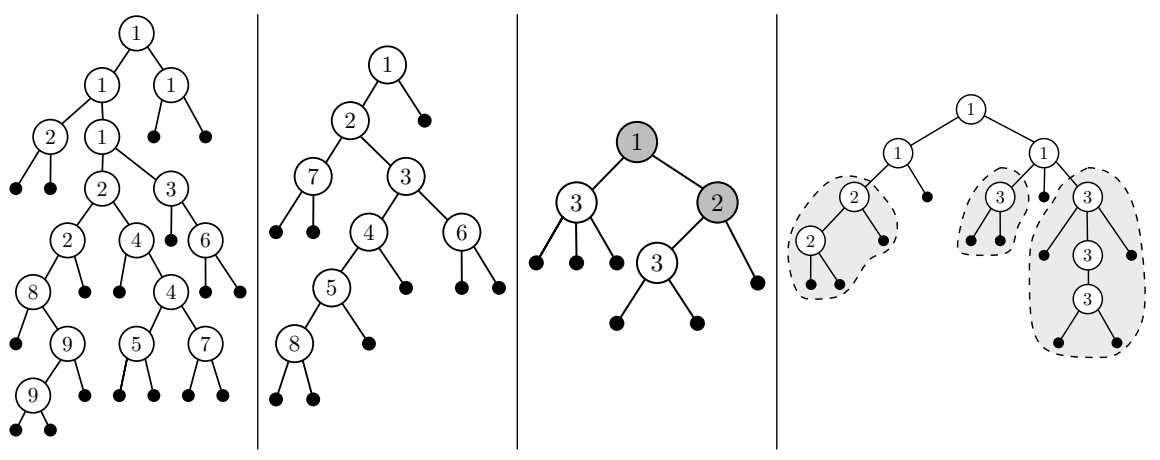

Fig. 1: (first) A monotonic binary tree of size 16; (second) a strictly monotonic binary tree of size 9 ; (third) a strictly monotonic tree of size 6 , with $\phi(z)=$ $z+2 z^{2}+2 z^{3}$; (fourth) a monotonic 2-3 tree of size 11: highlighted sub-trees shapes are the substitution of some step in the evolution process

Translating the above process using the framework of the symbolic method (see [7]), we obtain the following functional relation for the ordinary generating function $B$ enumerating trees built via the evolution process based on a degree function $\phi$ :

$$
B(z)=z+B(\phi(z))-B\left(\phi_{1} z\right) .
$$

Alternatively, the aforementioned evolution process may be expressed in terms of a function equation for $B(z)$ as

$$
B(z)=z+\sum_{n=1}^{\infty} \frac{z^{n}}{n !} B^{(n)}(z)(\phi(z)-z)^{n} .
$$

Where $B^{(k)}(z)$ is the $k$-th derivative of $B(z)$. The $n$-th term of the sum represents the process of pointing at $n$ leaves and substituting each by an element of the class represented by $\phi$. Note that the order in which we choose the leaves is irrelevant and so we divide by $n$ !.

Remark 2. This last formulation with a sum works when $\phi_{1}=1$ (i.e there are no unary nodes allowed).

The process is defined for $\phi_{1} \geq 1$, but it is worth noting that when $\phi_{1}=0$ we get families of balanced trees (all the leaves are at the same level). Meanwhile when $\phi_{1}=1$ we have trees with no unary nodes. Finally when $\phi_{1}>1$ we have $\left(\phi_{1}-1\right)$ colors for unary nodes. More details will be given in Section 3 . The generating series solution of the above functional relation are invariably purely formal, having radius of convergence equal to zero.

See Fig. 1 (first) and (second) for examples of, respectively a monotonic and a strictly monotonic binary tree.

We partition our analysis in two parts according to the value $\phi_{1}$ of the degree function. Let $B_{n}^{\phi}$ be the number of trees of size $n$ built via the evolution process. 


\begin{tabular}{lcccc}
\hline & $\phi_{1}$ degree function & Asymptotics & Ref. \\
\hline 2-3 Trees & 0 & $\phi(z)=z^{2}+z^{3}$ & $\Omega(\ln n) \frac{\phi^{n}}{n}$ & {$[14],[7]$} \\
\hline S. M. Binary Trees & 1 & $\phi(z)=z+z^{2}$ & $\alpha(n-1) ! n^{-\ln 2}\left(\frac{1}{\ln 2}\right)^{n}$ Thm $[1]$ \\
\hline S. M. General Schröder Trees & 2 & $\phi(z)=\frac{z}{1-z}+z$ & $\beta(n-1) ! 2^{\frac{(n-1)(n-2)}{2}}$ & Thm $[2]$ \\
\hline
\end{tabular}

Fig. 2: Some known combinatorial classes specifiable via equation (1), with different $\phi_{1}$ which results in different asymptotic scales. The function $\Omega$ is a periodic function with mean $(\phi \ln (4-\phi))^{-1}$ and $\phi=\frac{1+\sqrt{5}}{2}$. Here "S. M." stands for "Strictly Monotonic".

Theorem 1. Let $\phi(z)$ be such that $\phi_{1}=1, \phi_{2} \geq 1$ and $\forall i \geq 3, \phi_{i} \leq i \phi_{i-1}$. Then

$$
B_{n}^{\phi} \sim \kappa_{\phi}(n-1) !\left(\frac{\phi_{2}}{\ln 2}\right)^{n} n^{\left(-1+\frac{\phi_{3}}{\phi_{2}{ }^{2}}\right) \ln 2} .
$$

Theorem 2. Let $\phi(z)$ be such that $\phi_{1}>1, \phi_{2} \geq 1$ and $\forall i \geq 3, \phi_{i} \leq i \phi_{i-1}$. Then

$$
B_{n}^{\phi} \sim \kappa_{\phi} \phi_{1}^{\frac{(n-1)(n-2)}{2}}(n-1) ! \phi_{2}^{n} .
$$

In both cases $\kappa_{\phi}$ is a positive constant defined through an implicit equation.

It is of interest to note that the presence (or not) of unary nodes radically affects the asymptotic regime and that the first-order behavior of the asymptotics depends only on the first few terms of the degree function $\phi(z)\left(\phi_{2}\right.$ and $\phi_{3}$ for the first case and $\phi_{1}, \phi_{2}$ for the second). Finally the technical conditions $\phi_{i} \leq i ! \phi_{2}$ are not sharp but they are good enough for all practical applications.

As mentioned above, our scheme encompasses some classical tree models. For instance the models of balanced 2-3 trees and their generalization, the 2-3-4 trees, are obtained with $\phi(z)=z^{2}+z^{3}$ and $\phi(z)=z^{2}+z^{3}+z^{4}$ respectively. Note in both cases we have $\phi_{1}=0$. The model of $2-3$ trees has been introduced by Hopcroft as an efficient data structure and their asymptotics enumeration was given by Odlyzko [14. In fact when $\phi_{1}=0$ it is possible to obtain the exponential growth of these structures by computing the fixed point of $\phi(z)$, after which the asymptotic enumeration can usually be obtained by means of singularity analysis. In this paper we are interested in the cases where $\phi_{1}>0$, which leads to different asymptotic regimes, see Fig. 2.

\section{Applications}

We now exhibit examples of asymptotic enumeration for a number of interesting combinatorial structures. These are a direct application of the results presented in this paper.

\section{Strictly Monotonic Trees}

Example 1. Consider the class $\mathcal{T}$ with $\phi(z)=z+z^{2}+z^{3}$. The first few values of $\mathcal{T}_{n}$ are:

$$
0,1,1,3,12,68,482,4122,41253,472795, \ldots
$$




\begin{tabular}{llll}
\hline & $\phi(z)$ & Asymptotics & Ref. \\
\hline S. M. Binary Trees & $z+z^{2}$ & $\alpha(n-1) ! n^{-\ln 2}\left(\frac{1}{\ln 2}\right)^{n}$ & Thm 1$]$ \\
\hline S. M. Binary-Ternary Trees & $z+z^{2}+z^{3}$ & $\kappa(n-1) !\left(\frac{1}{\ln 2}\right)^{n}$ & Thm 1 \\
\hline S. M. Schröder trees & $\frac{z}{1-z}$ & $\beta(n-1) !\left(\frac{1}{\ln 2}\right)^{n}$ & Thm 1 [5] \\
\hline
\end{tabular}

Fig. 3: An example of the change in behavior of the asymptotics for different classes of our model. Here "S. M." stands for "Strictly Monotonic".

Asymptotically by Theorem 1, we have $\mathcal{T}_{n} \sim \kappa(n-1)$ ! $\left(\frac{2}{\ln 2}\right)^{n}$ with $\kappa \approx 0.41$.

This example is of interest as it indicates where the change of asymptotic behavior occurs when one varies the allowed node arities, going from binary trees with label repetitions (see [4]) to weakly increasing Schröder trees (see [5]) (with arbitrary arity of nodes).

The above example shows that the addition of just $z^{3}$ to $\phi(z)=z+z^{2}$ already results in a vanishing of the polynomial factor. Therefore adding higher powers of $z$ to $\phi(z)$ only affects the constant term. See also Fig. 3 As a further example, consider the class of such trees having binary nodes of two colors and ternary nodes again of two colors (see Fig. 1 (third)). By straightforward application of Theorem 2 we obtain the following.

Example 2. Consider the class $\mathcal{T}$ with $\phi=z+2 z^{2}+2 z^{3}$. The first few values of $\mathcal{T}_{n}$ are:

$$
0,1,2,10,76,804,10800,176240,3384176,74744016,1866432032 \ldots
$$

Asymptotically, we have that, $\mathcal{T}_{\phi, n} \sim \kappa(n-1) !\left(\frac{2}{\ln 2}\right)^{n} n^{\frac{-\ln 2}{2}}$ with $\kappa \approx 0.27$.

\section{Monotonic Trees}

Let $\mathcal{T}$ be some family of unlabeled rooted plane trees. We will denoted by $\mathcal{M T}$ the corresponding family of monotonic trees, i.e trees in $\mathcal{T}$ that have been labeled according to the rules for monotonic trees.

For example, consider the class of monotonic binary-ternary trees (see Fig. 1 (fourth)).

Example 3. Consider the class $\mathcal{B} \mathcal{T}$ of rooted plane binary-ternary unlabeled trees (whose size is their number of leaves). The specification of this class is

$$
\mathcal{B} \mathcal{T}=\mathcal{Z}+\operatorname{Seq}_{\{2,3\}} \mathcal{B T}
$$

where the first terms are $B T(z)=z+z^{2}+3 z^{3}+10 z^{4}+38 z^{5}+154 z^{6}+\ldots$ Then the first few values of $\mathcal{M B} \mathcal{T}_{n}$, i.e., the number of monotonic binary-ternary trees with $n$ leaves, are

$$
0,1,1,5,32,252,2340,25048,303862,4121730, \ldots
$$


By applying Theorem 1, $\mathcal{M B T}_{n} \sim \kappa(n-1) !\left(\frac{1}{\ln 2}\right)^{n} n^{2 \ln 2}$ with $\kappa \approx 0.17$.

Example 4. Let $S$ be the class of Schröder trees (all arities except unary are allowed) which has the following specification,

$$
\mathcal{S}=\mathcal{Z}+\operatorname{Seq}_{\geq 2} \mathcal{S}
$$

By solving the above equation, we have $S(z)=\frac{1}{4}\left(1+z-\sqrt{1-6 z+z^{2}}\right)$. The first terms of $S(z)$ are $z+z^{2}+3 z^{3}+11 z^{4}+45 z^{5}+197 z^{6}+\ldots$ Hence, the first values of $\mathcal{M S}_{n}$, i.e., the number of monotonic Schröder trees with $n$ leaves, are

$$
0,1,1,5,33,265,2497,27017,330409,4510065, \ldots
$$

\begin{tabular}{|c|c|c|c|}
\hline & $\phi$ & Asymptotics. & Refs. \\
\hline Strongly Increasing Schröder & - & $\frac{n !}{2}$ & [5] \\
\hline Strictly Monotonic Schröder & $\frac{z}{1-z}$ & $\alpha(n-1) !\left(\frac{1}{\ln 2}\right)^{n}$ & Thm 15 \\
\hline Monotonic Schröder & $S(z)$ & $\beta(n-1) !\left(\frac{1}{\ln 2}\right)^{n} n^{2 \ln 2}$ & Thm 1 \\
\hline
\end{tabular}

By Theorem 1 we have $\mathcal{M S}_{n} \sim \kappa(n-1) !\left(\frac{1}{\ln 2}\right)^{n} n^{2 \ln 2}$ with $\kappa \approx 0.19$.

Fig. 4: Comparison of the asymptotic behavior of increasing Schröder trees (where strongly increasing Schröder trees are increasing Schröder without label repetitions).

\section{Monotonic M-ary Trees}

It is a fact that our specification, by construction, enumerates families of trees by number of leaves. However there exists a special case, that of monotonic mary trees $(\mathcal{M T}$ where $\mathcal{T}$ is a variety of rooted plane $m$-ary trees $)$, where our specification also allows for enumeration by number of internal nodes. In this specific case then, we are also able to enumerate by number of internal nodes since any $m$-ary tree with $k$ leaves has $(k-1) /(m-1)$ internal nodes.

As an example, we consider the case of monotonic binary trees (see for example Fig. 1. In this case we obtain the following.

Example 5. Let $C$ be the class of plane binary trees with size equal to the number of leaves, given by $\mathcal{C}=\mathcal{Z}+\mathcal{C}^{2}$. These are counted by shifted Catalan numbers. By solving the above equation we find that $C(z)=\frac{1-\sqrt{1-4 z}}{2}$. Then the first few values of $\mathcal{M C}_{n}$, i.e the number of monotonic binary trees with $(n-1)$ internal nodes and $n$ leaves, are

$$
0,1,1,4,22,152,1264,12304,137332,1729584, \ldots
$$

By Theorem 1, we have that $\mathcal{M S}_{n} \sim \kappa(n-1) !\left(\frac{1}{\ln 2}\right)^{n} n^{\ln 2}$ with $\kappa \approx 0.34$. 


\begin{tabular}{llll}
\hline & $\phi$ & Asymptotics. & Ref. \\
\hline Increasing Binary Trees & - & $(n-1) !$ & {$[7]$} \\
\hline Strictly Monotonic Binary Trees & $z+z^{2}$ & $\alpha(n-1) !\left(\frac{1}{\ln 2}\right)^{n} n^{-\ln 2}$ & Thm 1 \\
\hline Monotonic Binary Trees & $C(z)$ & $\beta(n-1) !\left(\frac{1}{\ln 2}\right)^{n} n^{\ln 2}$ & Thm 1 \\
\hline
\end{tabular}

Fig. 5: Comparison of the asymptotic behavior between 3 classes of increasing binary trees.

\section{Combinatorial model and asymptotic analysis}

Using the above functional equation we can directly obtain the following recurrence:

$$
\begin{aligned}
& B_{1}=1, \\
& B_{n}=\sum_{k=1}^{n-1}\left(\sum_{i=1}^{n-k} \phi_{1}^{n-k-i}\left(\begin{array}{c}
n-k \\
i
\end{array}\right)\left[z^{k+i}\right]\left(\phi(z)-\phi_{1} z\right)^{i}\right) B_{n-k} .
\end{aligned}
$$

Let us define $T_{n}(n-k)=\sum_{i=1}^{n-k} \phi_{1}^{n-k-i}\left(\begin{array}{c}n-k \\ i\end{array}\right)\left[z^{k+i}\right]\left(\phi(z)-\phi_{1} z\right)^{i}$. We can then rewrite $B_{n}=\sum_{k=1}^{n-1} T_{n}(n-k) B_{n-k}$. In essence the coefficients of $T_{n}(n-k)$ represent the number of different combinations for a tree of size $n-k$ to be made into a tree of size $n$ by a subset of leaves into internal nodes that contains new leaves. The recurrence in equation (2) can be used to iterate on the specification and get the first few coefficients for a combinatorial class. It is also possible to write another recurrence for $B_{n}$ which involves sums over integer partitions.

\section{Proof sketch of Theorem 1}

The evolution process, cf. equation (1), that we study translates to generating functions which has a null convergence radius. Therefore its study needs a more elaborate approach. We present here a summary of our approach for the proof in 6 points:

1. Performing a Borel transform by rescaling coefficients by $n$ !.

2. Exhibit the dominant coefficients in the rescaled recurrence.

3. Find two new recurrences for the upper bound and the lower bound of $b_{n}$.

4. Write a new recurrence for the rescaled coefficients with a remainder term.

5. Deduce from it a linear differential equation.

6. Deduce the asymptotic behavior of the differential equation and determine the growth conditions on $\phi(z)$ necessary for the asymptotic to hold.

Let us carry on with the aforementioned plan, by first defining the following rescaled version of $B_{n}$ :

$$
b_{n}=\frac{B_{n}}{n !}
$$

From this we see that $B_{n}=n ! b_{n}$. Formally, this gives the following,

$$
b_{n}=\sum_{k=1}^{n-1} t_{n}(n-k) b_{n-k}
$$


where $t_{n}(i)$ is a sum of terms representing the different ways for a tree of size $i$ to be made into a tree of size $n$ by expanding some leaves into internal nodes with new leaves which all get the same label. These terms are each multiplied by $\frac{k !}{n !}$ due to the transform we have just performed. We notice also that the result are of power $j \leq 0$ in terms of $n$. To wit, the first few terms $T_{n}(n-1), T_{n}(n-2)$ transform (under Borel transforms) to $t_{n}(n-1), t_{n}(n-2)$, as follows:

$$
\begin{aligned}
T_{n}(n-1) B_{n-1}= & \phi_{2}(n-1) B_{n-1} \stackrel{\text { Borel }}{\longrightarrow} \phi_{2}(n-1) \frac{(n-1) !}{n !} b_{n-1}=t_{n}(n-1) b_{n-1} \\
T_{n}(n-2) B_{n-2}= & {\left[\frac{\phi_{2}^{2}(n-2)(n-3)}{2}+\phi_{3}(n-2)\right] B_{n-2} } \\
& \stackrel{\text { Borel }}{\longrightarrow}\left[\frac{\phi_{2}^{2}(n-2)(n-3)}{2 n(n-1)}+\frac{\phi_{3}(n-2)}{n(n-1)}\right] b_{n-2}=t_{n}(n-2) b_{n-2}
\end{aligned}
$$

The coefficients $t_{n}(n-k)$ are sums whose terms look like $f_{n}(\phi, k) \frac{p_{k}(n)}{n(n-1) \ldots(k+1)}$ where $p$ is a polynomial in $n$ of order at most $k$ and $f$ is a function which includes a ratio between the product of of elements of $\phi(z)$ divided by some factorial of $k$.

The highest order polynomial is of order $k$ and appears in the case where all leaves were replaced with binary nodes. In this case the corresponding term of $t_{n-k}$ is $\frac{\phi_{2}^{k}(n-k) \ldots(n-2 k+1)}{k ! n(n-1) \ldots(n-k+1)}$ which is of power 0 in $n$, as can be seen in the above example.

Our method of determining the dominant coefficients is based on a combinatorial argument. Nodes of lowest degree are the ones that count most, as the tree will have many permutations to create a lot of other trees of the same size.

Proposition 1. For $k \in\{1, \ldots, n-1\}$ the terms in $t_{n}(n-k)$ of order 0 in $n$ are

$$
\frac{\phi_{2}^{k}(n-k)}{k ! n}
$$

Proof. From the discussion above, the term of highest order in $n$ is $t_{n}(n-k)$ is (for $\left.k \in\left\{\left\lceil\frac{n}{2}\right\rceil, \ldots, n-1\right\}\right)$ :

$$
\frac{\phi_{2}^{k}(n-k) \ldots(n-2 k+1)}{k ! n(n-1) \ldots(n-k+1)} .
$$

It is then possible to factor out the desired term by making a polynomial division with two polynomials having the same order. The quotient is equal to 1 and we have a remainder term.

Proposition 2. For $k \in\{2, \ldots, n-1\}$ the terms in $t_{n}(n-k)$ of order $n^{-1}$ are the following

$$
\frac{\phi_{2}^{k-2} \phi_{3}}{(k-1) !(n-k+1)}-\frac{\phi_{2}^{k}}{(k-2) !(n-k+1)} .
$$

Proof. The term of second highest order in $n$ is $t_{n}(n-k)$ is:

$$
\frac{\phi_{2}^{k-1} \phi_{3}(n-k) \ldots(n-2 k+2)}{(k-2) ! n(n-1) \ldots(n-k+1)} \quad\left(\text { for } k \in\left\{\left\lfloor\frac{n}{2}\right\rfloor, \ldots, n-2\right\}\right) \text {. }
$$


We should also take the second order term of $\frac{\phi_{2}^{k}(n-k) \ldots(n-2 k+1)}{k ! n(n-1) \ldots(n-k+1)}$ into account, since it involves a term of order -1 in $n$.

From here by reasoning on the recurrence relation it is possible to conclude:

Proposition 3. Under the conditions of Theorem 1 on $\phi(z)$, the following holds:

$$
b_{n}=\Theta\left(\left(\frac{\phi_{2}}{\ln 2}\right)^{n} n^{\left(-1+\frac{\phi_{3}}{\phi_{2}^{2}}\right) \ln 2}\right) .
$$

Proof. The proof can be made upon finding upper and lower bounds on the coefficients $t_{n}(k)$, and translate the results to linear differential equations from which asymptotic behavior can be determined.

The last result does not give the asymptotic equivalent of the first order. For now we do not know if the function oscillates or not. We can write a new recurrence for $b_{n}$

$$
\begin{aligned}
b_{n}= & \sum_{k=2}^{n-1}\left(\frac{\phi_{2}^{k}}{k !}+\frac{\phi_{2}^{k-2} \phi_{3}}{(k-1) !(n-k+1)}-\frac{\phi_{2}^{k}}{(k-2) !(n-k+1)}\right) b_{n-k} \\
& +\phi_{2} b_{n-1}+a_{n} .
\end{aligned}
$$

where $a_{n}$ groups all omitted terms.

We can now determine the linear differential equation satisfied by $b(z)$, using the above recurrence, in which the coefficients transform into corresponding terms of the differential equation by simple manipulations as follows

$$
\begin{aligned}
\frac{\phi_{2}^{k}(n-k)}{k ! n} b_{n} & \rightarrow \frac{\phi_{2}^{k}}{k !} \int_{0}^{z} z^{k} b^{\prime}(z) d z ; \\
\frac{\phi_{2}^{k-2} \phi_{3}}{(k-1) !(n-k+1)} b_{n} & \rightarrow \frac{\phi_{2}^{k-2} \phi_{3} z^{k-1}}{(k-2) !} \int_{0}^{z} b(z) d z ; \\
\frac{-\phi_{2}^{k}}{(k-2) !(n-k+1)} b_{n} & \rightarrow \frac{-\phi_{2}^{k} z^{k-1}}{(k-2) !} \int_{0}^{z} b(z) d z ; \quad \text { and } a_{n} \text { translates into } a(z) .
\end{aligned}
$$

From the above we can derive the following integral form for $b(z)$

$$
b(z)=\int_{0}^{z}\left(e^{\phi_{2} z}-1\right) b^{\prime}(z) d z+\left(z \phi_{3}-{\phi_{2}}^{2} z\right) e^{\phi_{2} z} \int_{0}^{z} b(z) d z+a(z) .
$$

By differentiating the latter equation once we obtain

$$
\left(e^{\phi_{2} z}-2\right) b^{\prime}(z)+\left(z \phi_{3}-\phi_{2}^{2} z\right) e^{\phi_{2} z} b(z)+\tilde{a}(z)
$$

where $\tilde{a}(z)=a^{\prime}(z)+\left(\left(z \phi_{3}-\phi_{2}^{2} z\right) e^{\phi_{2} z}\right)^{\prime} \int_{0}^{z} b(z) d z$. Note that the second term in $\tilde{a}(z)$ is of smaller order than the first two terms of equation (4). 
Our problem has been transformed now into a more classical one where we have a linear differential equation with a regular singularity. The asymptotic behavior can be found by applying some classical theorems with some additional computations. The generic solution to the related homogeneous differential equation

$$
\left(e^{\phi_{2} z}-2\right) y^{\prime}(z)+\left(z \phi_{3}-{\phi_{2}}^{2} z\right) e^{\phi_{2} z} y(z)=0
$$

is $y(z)=C g(z)$ with $g(z)$ as follows

$$
g(z)=C \cdot\left(2-e^{\phi_{2} z}\right)^{\ln 2\left(\frac{\phi_{2}^{2}-\phi_{3}}{\phi_{2}^{2}}\right)} e^{-\left(\frac{\phi_{2}^{2}-\phi_{3}}{\phi_{2}^{2}}\right)\left((\ln 2)^{2}+L i_{2}\left(\frac{\exp \left(\phi_{2} z\right)}{2}\right)\right)},
$$

where the function $L i_{2}(z)$ stands for the dilogarithm function. Then, by variation of constants we obtain $C^{\prime}(z) \cdot \exp \left(\phi_{2} z-2\right) g(z)=\tilde{a}(z)$ and hence, as $b_{0}$ is 0 ,

$$
b(z)=g(z) \int_{0}^{z} \frac{\tilde{a}(t)}{\left(e^{\phi_{2} t}-2\right) g(t)} d t .
$$

In the following all the constants are positive. By the theory of complex linear differential equations studied in [9] and [16] and a good summary of the theorems in [7] we can deduce that $y(z)$ has a regular singular point at $z=\frac{\ln 2}{a}$, around it can be expanded as

$$
y(z) \underset{z \rightarrow \frac{\ln 2}{\phi_{2}}}{\sim} \kappa^{\prime}\left(z-\frac{\ln 2}{\phi_{2}}\right)^{\left(1-\frac{\phi_{3}}{\phi_{2}}\right) \ln 2}, \text { for some constant } \kappa^{\prime} .
$$

Therefore the expansion of $g(z)$ satisfies

$$
g(z) \underset{z \rightarrow \frac{\ln 2}{\phi_{2}}}{\sim} \kappa\left(z-\frac{\ln 2}{\phi_{2}}\right)^{\left(1-\frac{\phi_{3}}{\phi_{2}}\right) \ln 2}, \quad \text { for some constant } \kappa .
$$

Now that we have the singular expansion of $g(z)$. We need to understand how does the integral $\int_{0}^{z} \frac{\tilde{a}(t)}{\left(e^{\phi_{2} t}-2\right) g(t)} d t$ affects the main order asymptotic. And it will turn out that these integral is bounded depending on the growth of the coefficients of $\phi(z)$ as stated in the Theorem 1 .

Proposition 4. If the integral $\int_{0}^{z} \frac{\tilde{a}(t)}{\left(e^{\phi_{2} t}-2\right) g(t)} d t$ is bounded as $z \rightarrow \frac{\ln (2)}{\phi_{2}}$, then

$$
b_{n} \sim \alpha\left(\frac{\phi_{2}}{\ln 2}\right)^{n} n^{-1+\left(-1+\frac{\phi_{3}}{\phi_{2}{ }^{2}}\right) \ln 2} \quad \text { with } \quad \alpha=\kappa \int_{0}^{\frac{\ln 2}{\phi_{2}}} \frac{\tilde{a}(t)}{\left(e^{\phi_{2} t}-2\right) g(t)} d t
$$

The proof is based on the coefficients of the Cauchy product of $g(z)$ and the integral. We end the proof with two lemmas which show under which conditions on $\phi(z)$ the integral is bounded 
Lemma 1. If $\phi(z)$ fulfills the conditions of Theorem 1 then $\tilde{a}_{n}=\mathcal{O}\left(\frac{g_{n}}{n^{\epsilon}}\right)$ which in turn implies that the integral $\int_{0}^{\frac{\ln 2}{\phi_{2}}} \frac{\tilde{a}(t)}{\left(e^{a t}-2\right) g(t)} d t$ is bounded.

Proof. For the first implication, the result follows from Proposition 3 and a subsequent estimate on $a_{n}$. For the second implication we use the following argument. Let us denote $\beta=\left(-1+\frac{\phi_{3}}{\phi_{2}^{2}}\right) \ln 2$. We notice that

$$
\frac{1}{\left(e^{a t}-2\right) g(t)} \underset{z \rightarrow \frac{\ln 2}{a}}{\sim} c\left(z-\frac{\ln 2}{a}\right)^{-\beta},
$$

for some constant $c$. Furthermore, the coefficients of $\tilde{a}(z)$ are bounded above by some $\bar{a}_{n}=\left[z^{n}\right] c^{\prime}\left(z-\frac{\ln 2}{a}\right)^{\beta-\epsilon}$, with some constant $c^{\prime}$. Finally, we get

$$
\left[z^{n}\right] \frac{\tilde{a}(t)}{\left(e^{\phi_{2} t}-2\right) g(t)} \underset{z \rightarrow \frac{\ln 2}{a}}{\sim} \mathcal{O}\left(n^{-\epsilon}\right)
$$

and therefore

$$
\left[z^{n}\right] \int_{0}^{\frac{\ln 2}{\phi_{2}}} \frac{\tilde{a}(t)}{\left(e^{\phi_{2} t}-2\right) g(t)} \underset{z \rightarrow \frac{\ln 2}{a}}{\sim} \mathcal{O}\left(n^{-\epsilon-1}\right) .
$$

From Lemma 1, if $\phi(z)$ fulfills the conditions then the integral is bounded and the result holds.

\section{Conclusion}

We have presented an evolution-process-based framework for specifying and counting families of increasing trees allowing for label repetitions and weaklyincreasing sequences of labels along branches. Specifically, we have shown that under most interesting cases only binary and ternary do count in the main order asymptotic (unary and binary if unary nodes are allowed).

In this paper we discuss the case, where the coefficients of the degree function grow less rapidly than the process itself. Therefore, an interesting direction to pursue would be to have a full characterization of the asymptotic behavior of these processes depending on $\phi(z)$ for the other two cases. This analysis naturally follows from further applying the notions we have presented in this work.

Furthermore, the tools developed in this work may provide a novel way to approach the study of other structures of interest, such as linear $\lambda$-terms (see [3]), whose specifications also make critical use of the composition operation.

Acknowledgment. We thank Stephan Wagner for a fruitful discussion about the relationship of an involved proof of this paper and the article [8]. Furthermore we are grateful for the anonymous reviewers whose comments and suggestions helped improving and clarifying this manuscript. 


\section{References}

1. Bergeron, F., Flajolet, P., Salvy, B.: Varieties of increasing trees. In: CAAP. pp. 24-48 (1992)

2. Blieberger, J.: Monotonically labelled Motzkin trees. Discrete Applied Mathematics 18(1), 9-24 (1987)

3. Bodini, O., Gardy, D., Gittenberger, B., Jacquot, A.: Enumeration of generalized BCI lambda-terms. Electr. J. Comb. 20(4), P30 (2013)

4. Bodini, O., Genitrini, A., Gittenberger, B., Wagner, S.: On the number of increasing trees with label repetitions. Discrete Mathematics p. 111722 (2019)

5. Bodini, O., Genitrini, A., Naima, M.: Ranked Schröder Trees. In: 2019 Proceedings of the Sixteenth Workshop on Analytic Algorithmics and Combinatorics (ANALCO). pp. 13-26. SIAM (2019)

6. Flajolet, P., Gourdon, X., Martínez, C.: Patterns in random binary search trees. Random Structures \& Algorithms 11(3), 223-244 (1997)

7. Flajolet, P., Sedgewick, R.: Analytic Combinatorics. Cambridge University Press (2009)

8. Genitrini, A., Gittenberger, B., Kauers, M., Wallner, M.: Asymptotic enumeration of compacted binary trees of bounded right height. Journal of Combinatorial Theory, Series A 172, 105177 (2020)

9. Henrici, P.: Applied and computational complex analysis, vol. 2. Pure and applied mathematics a Wiley-Interscience series of texts, monographs, and tracts, J. Wiley and Sons (1974)

10. Kirschenhofer, P.: On the average shape of monotonically labelled tree structures. Discrete Applied Mathematics 7(2), 161-181 (1984)

11. Mendez, M.A.: Combinatorial differential operators in: Faa di Bruno formula, enumeration of ballot paths, enriched rooted trees and increasing rooted trees. Tech. rep., Cornell University (2016)

12. Morris, K.: On parameters in monotonically labelled trees. In: Drmota, M., Flajolet, P., Gardy, D., Gittenberger, B. (eds.) Mathematics and Computer Science III. pp. 261-263. Birkhäuser Basel (2004)

13. Morris, K.: Contributions to the analysis of increasing trees and other families of trees. Ph.D. thesis, University of the Witwatersrand, Johannesburg, South Africa (2005)

14. Odlyzko, A.M.: Periodic oscillations of coefficients of power series that satisfy functional equations. Advances in Mathematics 44(2), 180-205 (1982)

15. Prodinger, H., Urbanek, F.J.: On monotone functions of tree structures. Discrete Applied Mathematics 5(2), 223-239 (1983)

16. Wasow, W.: Asymptotic expansions for ordinary differential equations. Pure and Applied Mathematics, Vol. XIV, Interscience Publishers John Wiley \& Sons, Inc., New York-London-Sydney (1965) 